\title{
Emotional Regulation and Executive Function Deficits in Unmedicated Chinese Children with Oppositional Defiant Disorder
}

\author{
Wenqing Jiang, Yan Li, Yasong Du ${ }^{凶}$, and Juan Fan \\ Department of Child \& Adolescent Psychiatry, Shanghai Mental Health Center, Shanghai Jiaotong University School of Medicine, Shanghai, China
}

\begin{abstract}
Objective This study aims to explore the feature of emotional regulation and executive functions in oppositional defiant disorder (ODD) children.

Methods The emotional regulation and executive functions of adolescents with ODD, as well as the relationship between the two factors were analyzed using tools including Adolescent Daily Emotional Regulation Questionnaire (ADERQ), Wisconsin Card Sorting Test (WCST) and Cambridge Neuropsychological Test Automated Battery (CANTAB), in comparison with attention deficit hyperactivity disorder (ADHD) children without behavioral problem and healthy children; the ADERQ assessed emotional regulation ability and others were used to assess executive function.

Results Compared to normal children, the ODD group displayed significant differences in the scores of cognitive reappraisal, rumination, expressive suppression, and revealing of negative emotions, as well as in the score of cognitive reappraisal of positive emotions. WCST perseverative errors were well correlated with rumination of negative emotions $(r=0.47)$. Logistic regression revealed that the minimum number of moves in the Stocking of Cambridge (SOC) test (one test in CANTAB) and negative emotion revealing, were strongly associated with ODD diagnosis.

Conclusion Children with ODD showed emotion dysregulation, with negative emotion dysregulation as the main feature. Emotion dysregulation and the lack of ability to plan lead to executive function deficits. The executive function deficits may guide us to understand the deep mechanism under ODD.

Psychiatry Investig 2016;13(3):277-287
\end{abstract}

Key Words Oppositional defiant disorder, Emotion regulation, Executive functions.

\section{INTRODUCTION}

Oppositional defiant disorder (ODD) is a behavioral disorder that is mainly characterized by disobedient, provocative, defiant, hostile behaviors towards authority figures. According to the Diagnostic and Statistical Manual of Mental Disorders (DSM-IV) diagnostic criteria, ODD occurs in about $6 \%$ of children. ${ }^{1}$ According to Cavanagh et al. ${ }^{2}$ study, ODD is better considered as a disorder of emotional regulation.

Brown $^{3}$ elaborated the executive functions of an individual, and divided them into six modules, including activation, focus, effort, emotion, memory, and action. Of those, the emo-

Received: February 24, 2015 Revised: May 15, 2015

Accepted: May 15, 2015 Available online: January 11, 2016

$\triangle$ Correspondence: Yasong Du, PhD

Department of Child \& Adolescent Psychiatry, Shanghai Mental Health Center, Shanghai Jiaotong University School of Medicine, No.600 Wanping Road, Shanghai 200030, China

Tel: +86-21-64387250-3420, Fax: +86-21-64387986, E-mail: nanzixie@126.com

(a) This is an Open Access article distributed under the terms of the Creative Commons Attribution Non-Commercial License (http://creativecommons.org/licenses/by$\mathrm{nc} / 3.0$ ) which permits unrestricted non-commercial use, distribution, and reproduction in any medium, provided the original work is properly cited. tion module refers to the capability of an individual to manage frustrations and to regulate emotions. Therefore, the ability to regulate emotions is considered an important part of executive functioning.

Attention deficit hyperactivity disorder (ADHD) is a common seen behavioral disorder, which have a high comorbidity rate with $\mathrm{ODD}{ }^{4}$ The fact that $\mathrm{ADHD}$ children also have executive function deficits has been widely recognized. ${ }^{5,6}$ However, studies of executive function deficits of ODD children often have led to differing conclusions, not considering about the emotional regulation capability of ODD children. Some studies have confirmed executive function deficits in children with ODD. Rhodes et al. ${ }^{7}$ confirmed the existence of working memory deficits, which was an important part of executive functions, in children with ODD, especially visual working memory deficits. Schoemaker et al. ${ }^{8}$ also found that children with ODD displayed suppression deficits and that suppression was related to motivation incentives. However, there are contrary findings. Barnett et al. ${ }^{9}$ argued that the executive functions of ADHD children were not affected by comorbid ODD, 
suggesting that the executive function deficits of ODD itself were not obvious. Shuai et al. ${ }^{10}$ even found that disruptive behavioral disorder disruptive behavioral disorder (DBD)/ADHD children exhibited less impaired executive function compared with ADHD only children. (Note that DBD contains oppositional defiant disorder and conduct disorder (CD) according to DSM-IV.) Shuai et al. therefore argued that executive function deficits of DBD children were due to ADHD, and that the DBD/ADHD children performed better than ADHD children in terms of suppression interference.

In the dual-pathway model of executive functions, attention, working memory, planning, and response inhibition belong to the category of cold executive functions, while emotional regulation is more associated with hot executive functions, which are related to neuropsychological processes such as motivations and emotions. ${ }^{11}$ As Schoemaker et al. ${ }^{8}$ pointed out, the response inhibition of ODD children was related to the individual's motivation, and so hot executive function deficits might also be important defects in ODD. So it raises to us that whether ODD patients have executive function defect including emotional regulation problem or not? Currently, there are very few systematic studies of executive functions, especially the hot executive functions, in ODD children.

In other hand, emotion dysregulation is emerging as one of the core contributors to ADHD, ${ }^{12}$ which has gradually attracted widespread attention, and even has become an important criterion for adult ADHD diagnosis. Also, ADHD is the most common seeing problem of ODD, which has also been regarded as suffering from executive function defect. Is emotion regulation and executive function defect of ODD associated with or independent of ADHD?

We hypothesized that ODD children's emotion dysregulation is part of executive function deficits. Children with ODD suffer from executive function impairment including insufficient capability of emotion regulation. The characteristics of emotion regulation in ODD children are different from it in ADHD children. In this current study, we evaluated the emotion regulation and executive functions of ODD children, compared it to pure ADHD children without conduct problem and normal developed children. The relationship between $\mathrm{EF}$ and emotional regulation are also explored.

\section{METHODS}

\section{Participants}

All the data of participants came from the DBD database. The database was set up from January 2012. We collected information from drug naive DBD patients, who met the DSMIV diagnosis criterion of ADHD, ODD, CD, referred from child and adolescent out-patients clinic department, Shang- hai mental health centre. Only the participants whose age $\geq 10$ years were included as members of study group. We excluded any child with bipolar disorder or psychotic disorders, according to the Schedule for Affective Disorders and Schizophrenia for School-Age Children/Present and Lifetime Version (K-SADS-PL), or those whose Wechsler Intelligence Scale for Children (WISC-IV) score was $<70$. Further, we excluded children with serious organic disease or psychiatric disorders (including organic mental disorders, schizophrenia, and personality disorders); with neurodegenerative disorders, traumatic brain injury or cerebrovascular disease; with severe heart, liver, kidney dysfunctions, and other major physical illness history; or with a history of drug dependence.

Although all the participants were asked to finish all the assessment, among all the subjects included in this study, seven participants haven't finished the CANTAB tests, because of a long time consumption, in which, one in $\mathrm{ODD} / \mathrm{ADHD}$ group, one in simple ODD group, three in pure ADHD group and two in normal developed group.

Finally, the ODD group consisted of 24 children (23 males and 1 female) in the age range of 10-14 years (mean age $12.38 \pm 1.73$ years), including seven ODD patients and 17 ODD/ADHD comorbidity patients. ODD participants were all out-patients of SMHC, children and adolescent apartment, from January 2012 to December 2013.

ADHD group consisted of 24 children (22 males and 2 female) in the age range of 10-14 years (mean age 12.17 \pm 1.95 years), including $21 \mathrm{ADHD}$ inattention-subtype patients, 1 ADHD hyperactivity/impulsive subtype and 2 ADHD mixed subtype patients. ADHD participants were all out-patients of SMHC, children and adolescent apartment, from January 2012 to December 2013, whose mean conduct PSQ factor scores $<2$.

To form a normal comparison group, we recruited students from the elementary and middle schools of Zhabei District and Hongkou District of Shanghai city. The normal group consisted of students from the first grade in elementary school to the sophomore level in middle school. We randomly selected individuals according to their students number from schools and excluded any students diagnosed with DBD (ODD, CD) and ADHD, according to DSM-IV diagnostic criteria. Only the students who had completed the psychological questionnaire, whose age $\geq 10$ years, were selected as control group in this study. Students whose mean conduct PSQ score $\geq 2$ were also excluded. Finally, the normal group included 36 children ( 27 males and 9 females; all right handed) in the age range of 10-14 years (mean age $12.91 \pm 1.41$ years).

These three groups of children did not show significant age differences $(\mathrm{F}=1.643, \mathrm{p}=0.200)$, and gender difference $\left(X^{2}=\right.$ $5.415, \mathrm{p}=0.066$; Fisher exact test). 
This study was conducted in accordance with the declaration of Helsinki. This study was conducted with approval from the Ethics Committee. Written informed consent was obtained from all participants' guardians.

\section{Research tools}

1) Common demographic data were surveyed, including: gender, age, education and so on. 2) Conners Parent Symptom Questionnaire (PSQ): The PSQ is primarily used to evaluate the symptom severity of children behavioral problems. The PSQ contains a total of 48 items, uses 1-4 four-category rating scales, and generates six subscale scores, including scores reflecting conduct problems, learning problems, psychosomatic disorders, plus indices of impulsivity-hyperactivity, anxiety, and hyperactivity. The higher the score the more severe the corresponding problem is. The PSQ had good reliability and validity in China, and thus could be used to evaluate Chinese children. 3) Adolescent Daily Emotion Regulation Questionnaire (ADERQ): The ADERQ contains 35 items (15 evaluating positive emotion regulation and 20 assessing negative emotion regulation). Categories of response include cognitive reappraisal, rumination, expression suppression, and revealing. ADERQ was developed from Gross's ${ }^{13}$ Emotion Regulation Questionnaire (ERQ) by incorporating some typical daily emotion-evoking situations those teenagers often faced, based on Gross's emotion regulation process model. The scale distinguishes between positive and negative emotion regulation of an individual, and divides each individual's emotion regulation into cognitive regulation and expressive regulation. Further, it further divides cognitive regulation into cognitive reappraisal and rumination, and expressive regulation into revealing and suppression. The scale has shown acceptable reliability and validity in China. 4) Wechsler Intelligence Scale for Children: We used the WISC-IV adapted for Chinese individuals. ${ }^{14}$ The WISC-IV yields a general ability index and a cognitive efficiency index, each in two parts, with the former including verbal comprehension and perceptual reasoning subscales and the latter including working memory and processing speed subscales. Verbal comprehension includes three subtests (similarities, vocabulary, and comprehension); perceptual reasoning includes three subtests (block design, picture concepts, and matrix reasoning); working memory includes two subtests (digit span and letter-number sequencing); processing speed includes two subtests (coding and symbol search). Digit span test were conducted both in forward order, as heard, and in reverse order. 5) Stroop color-word association test: It is a classical tool for measuring inhibitory/control capability. There are two tests in Stroop color-word association test in all. In each test, there is a special card above which there are 4 bar, 28 entries of each column of the word (112 words) composition. In test A, participants must read the card of different characters with the fastest speed (red, blue, green, brown). The test time is limited to 2 minutes. Test B: also known as "interference test". Participants must read the card of the color of the background with the fastest speed (red, blue, green, brown). The correct reading number in test $\mathrm{A}$ and test $B$ are measured. 6) Wisconsin Card Sorting Test (WCST): The WCST was conducted on a computer which presented a total of 128 displays (cards). Participants matched a shape, which appeared on the lower left corner of the screen, with four template cards (either a red triangle, two green stars, three yellow diamonds, or four blue circles) on the top of the screen. Matching rules included color match, shape match, and item number match. The computer randomly determined a matching rule. When a participant made 10 consecutive correct matches, the computer automatically changed the rule. When six types of matches or 128 cards were finished, the test ended. The scores generated by this test included categories completed, perseverative errors, perseverative responses, and conceptual level response. 7) Cambridge Neuropsychological Test Automated Battery (CANTAB): The CANTAB is mainly used to assess executive functions. ${ }^{15}$ Tests used in this study included Spatial Span (SSP), Spatial Working Memory (SWM), and Stocking of Cambridge (SOC). In the SSP test, participants attempted to memorize the order of spatial locations; degree of success indicated the individual's visuo-spatial memory span. The SWM test required an individual to memorize the spatial positions as they appeared on the screen, and assessed the individual's ability to memorize space locations and refresh spatial memory. The SOC evaluated the individual's capacity for planning and problem-solving, which asked individual to finish the task as rapidly as possible without making mistake. DMTs was also tested, but not included in this study.

\section{Survey procedure}

After parents signed informed consent, all the DBD participants attending our database of DBD, who met the inclusion criteria were tested with the Stroop color-word associated test, WISC-IV, the WCST, and then, the CANTAB to assess their executive functions. If the participants were 10 years or more, the ADERQ was asked to finished. Meanwhile the parents took a questionnaire survey, including general demographic information questionnaire and PSQ. In general the demographic information questionnaire, birth data of the child, gender, educational level of the child were collected.

The students in school, who met the inclusion criteria took neuropsychological tests and the ADERQ, while their parents took psychological questionnaires. We obtained conduct scores for participants in the normal group from the PSQs completed by participants' parents. We excluded students 


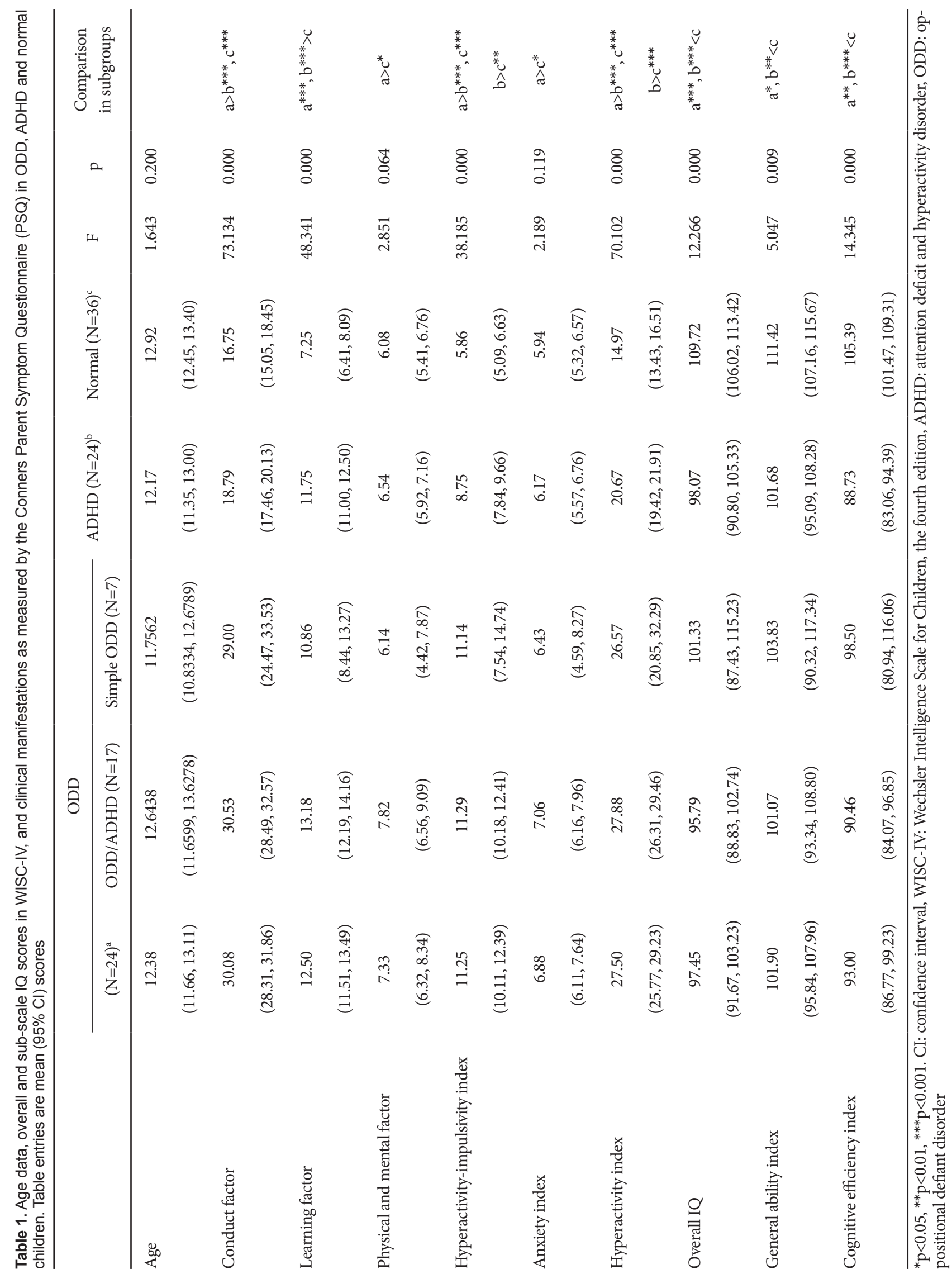


whose mean conduct problems scores were $\geq 2$.

\section{Statistical analysis}

All data were processed using SPSS11.5 to establish a database and statistical analysis. The statistical method comprised analysis of variance (ANOVA) and LSD t-test, in which mean values in three groups were compared when the data meet the normal distribution; others were compared using appropriate non-parametric tests (e.g. the M-W test) according to Kolmogorov-Smirnov test. Chi-square test was performed to the enumeration data. Pearson correlation and Spearman correlation test and logistic regression were also used to explore the risk factors of ODD and ADHD.

\section{RESULTS}

\section{Comparison of WISC-IV intelligence quotient}

Comparisons using ANOVA test showed that there were significant differences in the average IQ between the three groups of children (Table 1). As we can see, both ODD group and ADHD group performed worse than normally developed children in IQ performance and cognitive efficiency index scores. And ODD group performed worse than normally developed children in general ability index.

In two sample t-test, simple ODD subgroup is not significantly different from normal developed children in IQ performance, which maybe due to the small sample. Meanwhile, the ODD comorbid ADHD group showed significantly lower total IQ scores $[t(51)=3.928, p<0.001]$, general ability index scores $[\mathrm{t}(51)=2.436, \mathrm{p}=0.019]$, and cognitive efficiency index scores $[\mathrm{t}(51)=4.250, \mathrm{p}<0.001]$ than the normal group.

\section{Comparison of clinical symptoms according to PSQ}

Comparisons using ANOVA test showed that there were significant differences in the PSQ questionnaire between three groups of children (Table 1). Compared with the normal group (Table 1), the ODD group and ADHD group showed significantly higher scores on several PSQ factor than normally developed children in t-test, includes conduct factor, learning factor, hyperactivity-impulsivity index and hyperactivity index, which indicated that ODD and ADHD group participants suffer more behavioral problem and learning difficulties. ODD group also showed significant higher score than normal developed children in physical and mental factor and anxiety index.

In particular, the PSQ conduct factor score $[\mathrm{t}(51)=-5.704$, $\mathrm{p}=0.001]$, learning factor score $[\mathrm{t}(51)=-2.864, \mathrm{p}=0.007]$, physical and mental factor score $[Z(17,36)=3.505, p<0.001]$, hyperactivity factor score $[Z(17,36)=4.421, \mathrm{p}<0.001]$, anxiety factor score $[t(51)=-2.056, p=0.048]$, and hyperactivity index score 


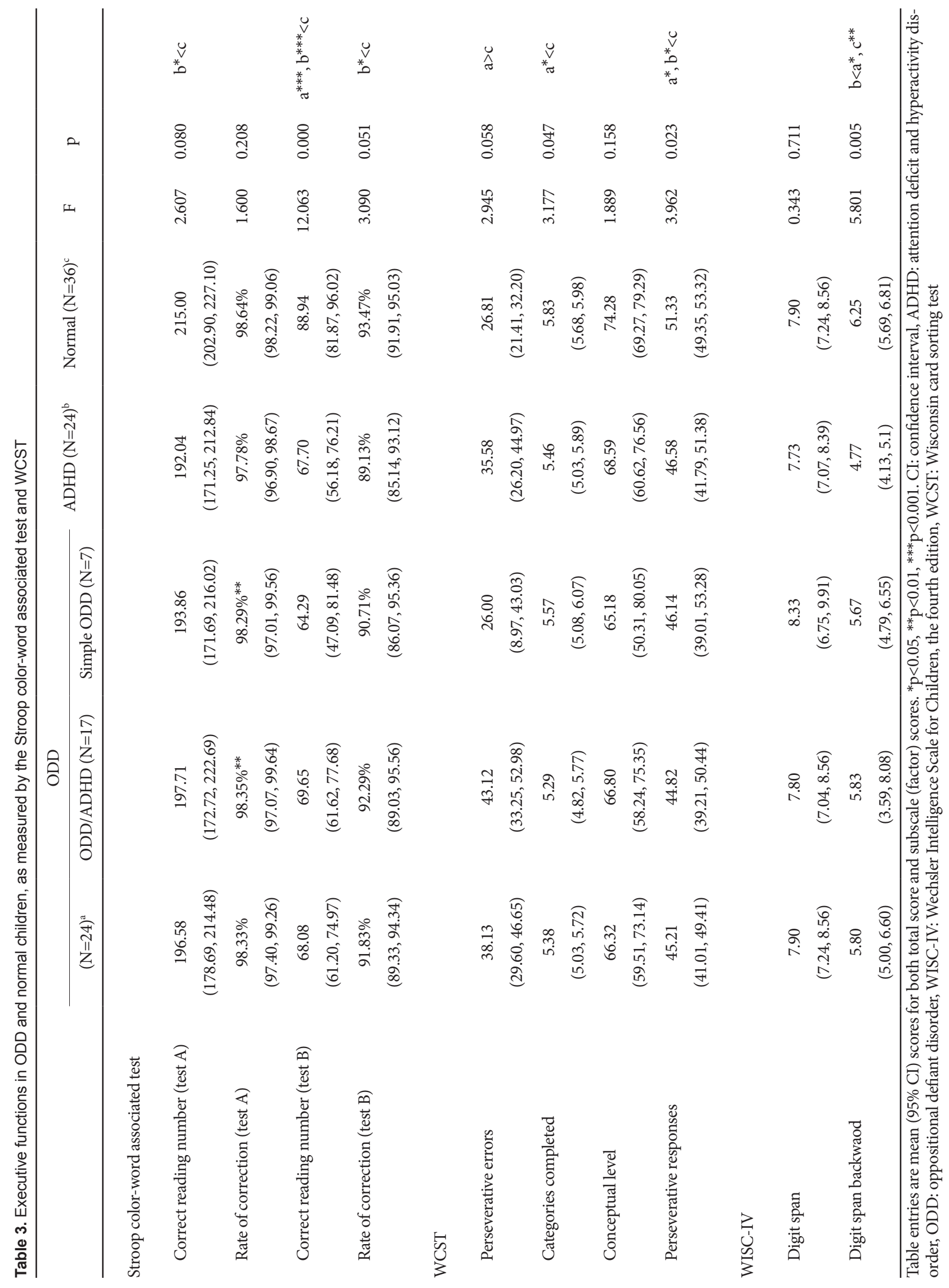


$[\mathrm{t}(51)=-5.011, \mathrm{p}<0.001]$ of the ODD comorbid ADHD group all were significantly higher than those of the normal group. Compared to the normal group, the simple ODD subgroup had significantly higher PSQ conduct factor scores [ $\mathrm{t}(41)=$ $-8.543, \mathrm{p}<0.001]$, learning factor scores [t $(41)=-7.338, \mathrm{p}<0.001]$, hyperactivity factor scores $[\mathrm{Z}(7,36)=2.704, \mathrm{p}=0.007]$ and hyperactivity factor scores $[\mathrm{t}(41)=-8.532, \mathrm{p}<0.001]$ according to the result of two sample t-test or M-W test. However, the simple ODD and ODD comorbid subgroups showed no significant differences in PSQ scores.

\section{Comparison of ADERQ (emotion regulation) scores}

Comparisons using ANOVA test showed that there were significant differences in the ADERQ questionnaire, including negative emotion cognitive reappraisal, negative emotion expressive suppression, negative emotion expressive revealing and positive emotion cognitive reappraisal between three groups of children (Table 2). When compared with the normal group, ODD group and ADHD group showed significant higher score in negative emotion expressive revealing and significant lower score in negative emotion cognitive reappraisal, negative emotion expressive suppression and positive emotion cognitive reappraisal with two sample t-test (Table 2). And ODD group showed significant higher score in negative emotion cognitive rumination than normal group.

In two sample test, the ODD comorbid ADHD group had significantly higher scores than the normal group on rumination $[\mathrm{t}(51)=-2.142, \mathrm{p}=0.044]$ and revealing $[\mathrm{t}(51)=-2.608$, $\mathrm{p}=0.018]$, but significantly lower scores on expressive suppression of negative emotions $[t(51)=2.198, p=0.033]$ according to the result of two sample t-test. The simple ODD subgroup had significantly lower scores on cognitive reappraisal of negative emotions than the normal group $[t(41)=2.081$, $\mathrm{p}=0.044]$. There were no significant differences between two ODD subgroups.

\section{Comparison of executive functions in Stroop color- word associated test, the WCST and WISC-IV}

Comparisons using ANOVA test showed that there were significant differences in several items of digital span in WISCIV, Stroop color-word associated test and WCST. ADHD group showed significant lower score in rate of correction in test $\mathrm{A}$, correct reading number in test $\mathrm{A}$ and test $\mathrm{B}$, while $\mathrm{ODD}$ group showed significant lower score in correct reading number in test B. Compared to normal group, ODD group showed significant higher score in perseverative errors and lower score in categories completed and perseverative responses.

In two sample $t$-test, the ODD comorbid ADHD group had significantly higher perseverative errors on the WCST $[t(50)=$ $-2.872, \mathrm{p}=0.006$, while completing a significantly different distribution in number of categories compared to the normal group (Table 3).

\section{Comparison of executive functions in CANTAB}

Comparisons using ANOVA test showed that there were significant differences in several items of CANTAB test, including SOC problems solved in minimum moves, SWM between errors, SWM strategy, SOC mean moves (Table 4).

Compared using two sample t-tests, the CANTAB SWM between errors $[t(51)=-2.413, p=0.020]$ in ODD/ADHD subgroup were significantly higher than those of the normal group, which meant that ODD/ADHD group participants made more errors in the spacial working memory task. Also SOC mean move [ $t(51)=-2.450, p=0.025]$ scores in ODD/ADHD subgroup were significantly higher than those of the normal group, which meant that ODD/ADHD group participants took more steps in finishing SOC task, while the SSP score $[\mathrm{t}(51)=$ $2.620, p=0.012]$ was significantly lower than those of the normal group, which indicated comparatively poor spatial working memory. Third, the SOC problems solved in minimum moves test were significantly lower than those of the normal group $[t(51)=3.975, p<0.001]$, which meant the pure ODD group performed worse in making plans. Compared to simple ODD group, ODD/ADHD subgroup had lower score in SSP test $[\mathrm{t}(20)=2.129, \mathrm{p}=0.042]$.

\section{Correlational analysis in ODD group}

We summarized the Pearson correlations between the main dependent variables in ODD group.

Pearson correlation analysis showed significant correlations between conduct PSQ factor score and negative emotion expressive suppression $(\mathrm{r}=0.412, \mathrm{p}=0.045)$ and positive emotion cognitive rumination $(\mathrm{r}=0.456, \mathrm{p}=0.025)$, which showed the relationship between behavior problem and emotion regulation.

Non-significant correlations have been found between emotion regulation and Stroop color-word associated test, digital test in WISC-IV, measure items in CANTAB. When the emotion regulation scores were tested with WCST, the results suggested that perseverative errors of WCST $(r=0.474, p=0.019)$ correlated well with negative emotion rumination. The relationship between executive function and emotional regulation may suggest the common neuropsychological basis of impulse.

\section{Logistic regression analysis}

We conducted Spearman correlation test and logistic regression analysis, trying to make out the risk factor for developing ODD and ADHD. In the result of Spearman correlation, it turned out that whether ODD diagnosis correlated with correct reading number of test $\mathrm{B}(\mathrm{r}=-0.478, \mathrm{p}=0.000)$, WCST perseverative errors $(r=0.294, p=0.023)$, categories 
completed $(\mathrm{r}=-0.357, \mathrm{p}=0.005)$, conceptual level $(\mathrm{r}=-0.261$, $\mathrm{p}=0.044)$, perseverative responses $(\mathrm{r}=-0.289, \mathrm{p}=0.025)$, the minimum moves of CANTAB SOC test $(r=-0.473, p<0.001)$, SOC mean moves (all steps) $(\mathrm{r}=0.320, \mathrm{p}=0.020)$, SSP span length ( $\mathrm{r}=-0.334, \mathrm{p}=0.013)$, SWM between errors $(\mathrm{r}=0.308$, $\mathrm{p}=0.021)$, SWM strategy $(\mathrm{r}=0.335, \mathrm{p}=0.012)$, negative emotion revealing $(\mathrm{r}=0.307, \mathrm{p}=0.017)$, and cognitive reappraisal of positive emotions $(\mathrm{r}=-0.270, \mathrm{p}=0.037)$.

Then we performed a logistic regression analysis, using associated factors in executive function test of WCST, CANTAB, and emotion regulation questionnaire in ADERQ as independent variables and ODD as the dependent variable. The results suggested that the minimum moves of the SOC test, correct reading number in test $\mathrm{B}$ and negative emotions revealing fitted the regression equation very well $\left(X^{2}=25.607\right.$, $\mathrm{p}=0.000$ ) (Table 5).

We also performed a logistic regression analysis, using associated factors in executive function test of WCST, CAN$\mathrm{TAB}$, and emotion regulation questionnaire in ADERQ as independent variables and $\mathrm{ADHD}$ as the dependent variable. In the result of Spearman correlation, it turned out that whether ADHD diagnosis correlated with correct reading number in test $\mathrm{A}(\mathrm{r}=-0.262, \mathrm{p}=0.045)$ and test $\mathrm{B}(\mathrm{r}=-0.413$, $\mathrm{p}=0.001)$, SOC problems solved in minimum moves $(\mathrm{r}=$ $-0.416, \mathrm{p}=0.002)$, SOC mean moves (all steps) $(\mathrm{r}=-0.371, \mathrm{p}=$ 0.007 ), word span backward ( $\mathrm{r}=-0.426, \mathrm{p}=0.001)$, cognitive reappraisal of negative emotions $(r=-0.487, \mathrm{p}<0.001)$, suppression of negative emotions $(\mathrm{r}=-0.548, \mathrm{p}<0.001)$, negative emotion revealing $(r=0.292, p=0.024)$, or cognitive reappraisal of positive emotions $(\mathrm{r}=-0.389, \mathrm{p}=0.002)$.

Then we performed a logistic regression analysis, using associated factors in executive function test of WCST, CANTAB, and emotion regulation questionnaire in ADERQ as independent variables and ADHD as the dependent variable. The results suggested that correct reading number in test $\mathrm{B}$, negative emotions revealing and expressive suppression fitted the regression equation very well $\left(\chi^{2}=42.583, p=0.000\right)$ (Table 5).

\section{DISCUSSION}

Eisenberg and Spinrad ${ }^{16}$ defined emotion regulation as "the process of initiating, avoiding, inhibiting, maintaining, or modulating the occurrence, form, intensity, or duration of internal feeling states, ... in the service of accomplishing affect-related biological or social adaptation or achieving individual goals". ODD is now considered as a type disease related to emotion dysregulation ${ }^{2}$ and the ability to regulate emotions is an important part of an individual's executive functions. ${ }^{3}$ Previous research ${ }^{17}$ suggested that executive function deficits may be more related to hot executive functions and hot execu- 
tive functions are related to motivation and emotion. But currently the characteristics of emotion dysregulation in ODD children are not clear. The effects of different dimensions of executive functions on ODD need further investigation, in particular, a combinatorial measurement of both hot and cold executive functions. Moreover, ODD is inextricably associated with the ADHD, the two have a high comorbidity rate, and there are also emotion regulation problems and executive function defects associated with ADHD. So, whether ODD children's emotion regulation problems are due to ADHD or are independent of ADHD is still unclear.

The results of this study showed that $70.8 \%$, of children diagnosed with ODD displayed ADHD comorbidity, and most of them were males. Children with ODD, had lower overall intelligence, a lower general cognitive ability index, and a lower cognitive efficiency index than normal children. Comorbid ADHD/ODD children's IQs were even lower. ODD children showed notable differences in clinical manifestations and development compared with healthy children, and exhibited general behavior problems, such as hyperactivity and impulsivity, as well as emotional problems. ${ }^{18}$ ODD children are prone to negative emotions, they are easily annoyed and tend to get angry and resentful, and they show more crying, sadness, and anxiety. ${ }^{19}$ Similarly, this study also found that ODD children more easily show anxiety than healthy children.

Leibenluft et al. ${ }^{20}$ brought out the concept of severe mood dysfunction (SMD) of children and adolescents. SMD has irritability and emotional instability as the main symptoms, and nearly a quarter of SMD children will develop ODD. ${ }^{21}$ All these studies strongly suggested that there were more sustained negative emotions and emotional instability in children with ODD.

But an unsolved problem is the feature of ODD children's emotional regulation. In our study, we found that ODD children showed significantly different feature of emotional regu- lation from healthy children and this difference was mainly in the regulation of negative emotions. Specifically, when ODD children had negative emotions (such as sadness, frustration, and anger), they tended to immerse themselves in their own emotions and were not good at self-regulation. They were less likely to divert their attention and they were not good at rethinking negative events and emotions to facilitate self-help. These children also were less good at suppressing or controlling their negative emotions, but easily expressed their displeasure, grievances, and anger, especially anger. This finding can better explain clinical manifestations in ODD children, who are more susceptible to be controlled by emotions. Once the negative emotions appeared, these children lacked effective regulation strategies and were not good at self-control, but tended to reveal their negative emotions, so that their behaviors were more impulsive and destructive, which is consistent to the finding of Boylan and Whelan.

Because emotion regulation may constitute a dimension of executive function, the present study also measured other executive functions in ODD children, and the relationship between traditional EFs tasks and emotional regulation were also explored. We found that children with ODD had executive function deficits in multiple dimensions including working memory, impulsiveness, and planning capabilities. ODD children with comorbid ADHD had more significant and widespread damage in executive functions, while the children diagnosed with simple ODD got lower scores on the SOC subscale of the minimum number of moves. SOC tests the ability of an individual to accomplish tasks with a minimum of steps through planning. The more steps are used by the individual, the worse the individual's ability to plan is. Therefore, the executive function deficits of ODD children in the clinic may be in a large part due to their comorbid $\mathrm{ADHD}$, which is consistent to the finding of Sjowall et al., ${ }^{22}$ while for the simple ODD child, the problem is mainly a lack of planning and proneness to impul-

Table 5. ODD and ADHD risk factors of executive functions and emotion regulation

\begin{tabular}{|c|c|c|c|c|c|}
\hline & B & SE & Wald & $\mathrm{p}$ & $\operatorname{Ext}(\mathrm{B})$ \\
\hline \multicolumn{6}{|l|}{ ODD } \\
\hline Correct reading number in test $\mathrm{B}$ & -0.056 & 0.024 & 5.369 & 0.020 & 0.946 \\
\hline SOC Problems solved in minimum moves & -0.367 & 0.192 & 3.666 & 0.056 & 0.693 \\
\hline Negative emotion expressive revealing & 0.368 & 0.161 & 5.210 & 0.022 & 1.445 \\
\hline Constant & 3.339 & 1.971 & 2.869 & 0.090 & 28.181 \\
\hline \multicolumn{6}{|l|}{ ADHD } \\
\hline Correct reading number in test $B$ & -0.089 & 0.039 & 5.272 & 0.022 & 0.915 \\
\hline Negative emotion expressive suppression & -0.989 & 0.376 & 6.904 & 0.009 & 0.372 \\
\hline Negative emotion expressive revealing & 0.847 & 0.327 & 6.711 & 0.010 & 2.332 \\
\hline Constant & 9.469 & 4.001 & 5.600 & 0.018 & 12954.174 \\
\hline
\end{tabular}

B: coefficient B, Wald: Wald Value, $\operatorname{Exp}(B)$ : OR 
sive behavior.

Emotional regulation is suspected as a part of EF. The relationship between EF and emotional regulation was explored. According to our result, emotional regulation of ODD children is not only related to their clinical manifestation, but also to the result of WCST. In ODD children, negative emotion rumination correlated with WCST perseverative errors. Perseverative errors are the numbers of the repetitions of wrong choices by the tested individuals. Perseverative errors indicate the capability of tested individuals to learn from their mistakes and therefore to change strategy. A greater number of perseverative errors suggest that the individual has self-reflection and selfcorrection defects, is prone to impulsive behavior, and is not good at thinking. Our results suggested that children with ODD were less likely to receive reminders to change their cognitive strategies, but more inclined to immerse themselves in their negative emotions, and that the characteristics exhibited at a cognitive level were consistent with the stubbornness displayed at emotional level.

In addition, the results of this study also suggested that, of all executive function indices measured in ODD children, correct reading number in test $\mathrm{B}$, SOC minimum number of moves, and negative emotion expressive revealing are the risk factors that collectively contribute to ODD. Response inhibition capability, planning dysfunction, and emotion dysregulation are important risk factors for ODD. In SonugaBarke et al. ${ }^{11}$ dual-pathway model, the individual's executive functions are divided into the traditional executive functions or cold executive functions, such as attention, working memory, planning, and inhibition, and hot executive functions which are related to neuropsychological processes such as emotion and motivation. The findings of our study demonstrated that executive dysfunction in ODD children may involve both cold and hot functions. Consistent with previous knowledge of ODD, van Goozen et al. ${ }^{17}$ pointed out that, although children with ODD had motivation-related suppression deficits, they also had hot executive function deficits. In Hobson et al. ${ }^{23}$ study, disruptive behavior disorder suffer from hot executive function deficits.

In the analysis of subgroups, the current study revealed that, regardless of ADHD comorbidity, ODD children always exhibited emotion dysregulation and this defect was more significant in ADHD/ODD children. So how are emotion regulation, $\mathrm{ADHD}$, and ODD connected? ODD and ADHD are considered as two different diseases with different pathogenesis, Forssman et al. ${ }^{24}$ suggested that ADHD behaviors were associated with cognitive function, while factors independent of cognitive function (e.g., environmental risk factors, including family environmental factors) were associated with ODD behaviors. ADHD is a kind of developmental dis- order. These core symptoms of ADHD were associated with brain development, consistent with their nature as developmental problems. In this study, we selected ADHD patients without conduct problem to avoid their overlap of behavior feature in clinic manifestation. The result shows that not only several executive function factors but also emotional regulation features are also risk factor in ADHD, which is consistent to the finding of emotional dysregulation in ADHD. Previous work $^{25}$ suggested that children with ADHD had emotion dysregulation, and comorbid ADHD externalizing and emotion dysregulation were more relevant. Unfortunately, our result of pure ODD children is still limited by the small sample. The role of emotion dysregulation in ADHD and ODD need to be further researched.

Currently ADHD children's emotion dysregulation receives more attention, but the current study demonstrated that emotion dysregulation also occurred in ODD children. However, this generalizability of the results of the present study is constrained by the relatively small sample size and cannot determine that ODD children's emotion dysregulation is independent of ADHD; therefore, further study of ADHD children's emotion regulation is needed to rule out this possibility. Whether ADHD and ODD children have the same underlying mechanisms of emotion dysregulation is worth further study.

\section{Limitation}

In this study, 70\% children were ODD/ADHD comorbidity patients, and therefore, the conclusion of the present study is mainly applied to children with both ADHD and ODD. Simple ODD children showed no significant differences in executive functions from normal children, but this may be related to the small sample size of simple ODD, thus future studies need to expand the sample size and analyze the simple ODD subgroups. In this study, data were collected through children self-reporting. Further tools in evaluating the emotional regulation should be introduced.

\section{Acknowledgments}

We thank Professor Alastair Vance from Melbourne Royal Children Hospital, who is so kind in helping introducing CANTAB to our department. We also thank Biao Sang and Xinmei Deng of East China Normal University for their helping with administration and scoring of the ADERQ. We thank Xiangyang Zhong of the Zhabei District Teacher Academy and Hongli Wang of the Hongkou District Teacher Academy for their help in data collection from the normal children.

This study was supported by a grant of the 2012 general program of National Natural Science Foundation of China (Grant Number: 81271510), a grant of 2012 Shanghai Municipal Health Bureau Youth Project (Grant Number: 20124y042), a 2011 Shanghai Mental Health Center hospital-level project (Grant Number: 2011-YJ-07), and 2013 Shanghai Mental Health Center featured specialty development project (Grant Number: 2013YJTSZK-04). 


\section{REFERENCES}

1. Merikangas KR, Nakamura EF, Kessler RC. Epidemiology of mental disorders in children and adolescents. Dialogues Clin Neurosci 2009; 11:7-20.

2. Cavanagh M, Quinn D, Duncan D, Graham T, Balbuena L. Oppositional defiant disorder is better conceptualized as a disorder of emotional regulation. J Atten Disord 2014 [Epub ahead of print].

3. Brown TE. Executive functions and attention deficit hyperactivity disorder: implications of two conflicting views. Int J Disab Develop Edu 2006;53:35-46.

4. Jensen CM, Steinhausen HC. Comorbid mental disorders in children and adolescents with attention-deficit/hyperactivity disorder in a large nationwide study. Atten Defic Hyperact Disord 2015;7:27-38.

5. Barkley RA. Behavioral inhibition, sustained attention and executive functions: constructing a unifying theory of ADHD. Psychol Bull 1997; 121:65-94.

6. Coghill DR, Seth S, Matthews K. A comprehensive assessment of memory, delay aversion, timing, inhibition, decision making and variability in attention deficit hyperactivity disorder: advancing beyond the three pathway models. Psychol Med 2014;44:1989-2001.

7. Rhodes SM, Park J, Seth S, Coghill DR. A comprehensive investigation of memory impairment in attention deficit hyperactivity disorder and oppositional defiant disorder. J Child Psychol Psychiatry 2012;53:128-137.

8. Schoemaker K, Bunte T, Wiebe SA, Espy KA, Deković M, Matthys W. Executive function deficits in preschool children with ADHD and DBD. J Child Psychol Psychiatry 2012;53:111-119.

9. Barnett R, Maruff P, Vance A. Neurocognitive function in attentiondeficit/hyperactivity disorder with and without comorbid disruptive behaviour disorders. Aust N Z J Psychiatry 2009;43:722-730.

10. Shuai L, Chan RC, Wang Y. Executive function profile of Chinese boys with attention-deficit hyperactivity disorder: different subtypes and comorbidity. Arch Clin Neuropsychol 2011;26:120-132.

11. Sonuga-Barke EJ. The dual pathway model of AD/HD: an elaboration of neuro-developmental characteristics. Neurosci Biobehav Rev 2003; 27:593-604.

12. Shaw P, Stringaris A, Nigg J, Leibenluft E. Emotion dysregulation in attention deficit hyperactivity disorder. Am J Psychiatry 2014;171:276-293.

13. Gross JJ. The emerging field of emotion regulation: an integrative re- view. Rev Gen Psychol 1998;2:271-299.

14. Li YQ. Structural Changes in the Fourth Edition of the Wechsler Intelligence Scale for Children and assessment of children's cognitive abilities. Educ Meas Eval 2009;4:4-7.

15. Gau SS, Shang CY. Executive functions as endophenotypes in ADHD: evidence from the Cambridge Neuropsychological Test Battery (CANTAB). J Child Psychol Psychiatry 2010;51:838-849.

16. Eisenberg N, Spinrad TL. Emotion-related regulation: sharpening the definition. Child Dev 2004;75:334-339.

17. van Goozen SH, Cohen-Kettenis PT, Snoek H, Matthys W, SwaabBarneveld H, van Engeland H. Executive functioning in children: a comparison of ospitalized ODD and ODD/ADHD children and normal controls. J Child Psychol Psychiatry 2004;45:284-292.

18. Boylan K, Vaillancourt T, Boyle M, Szatmari P. Comorbidity of internalizing disorders in children with oppositional defiant disorder. Eur Child Adolesc Psychiatry 2007;16:484-494.

19. Whelan YM, Stringaris A, Maughan B, Barker ED. Developmental continuity of oppositional defiant disorder subdimensions at ages 8,10 , and 13 years and their distinct psychiatric outcomes at age 16 years. J Am Acad Child Adolesc Psychiatry 2013;52:961-969.

20. Leibenluft E, Charney DS, Towbin KE, Bhangoo RK, Pine DS. Defining clinical phenotypes of juvenile mania. Am J Psychiatry 2003;160:430-437.

21. Brotman MA, Kassem L, Reising MM, Guyer AE, Dickstein DP, Rich BA, et al. Parental diagnoses in youth with narrow phenotype bipolar disorder or severe mood dysregulation. Am J Psychiatry 2007;164:1238-1241.

22. Sjöwall D, Roth L, Lindqvist S, Thorell LB. Multiple deficits in ADHD: executive dysfunction, delay aversion, reaction time variability, and emotional deficits. J Child Psychol Psychiatry 2013;54:619-627.

23. Hobson CW, Scott S, Rubia K. Investigation of cool and hot executive function in ODD/CD independently of ADHD. J Child Psychol Psychiatry 2011;52:1035-1043.

24. Forssman L, Eninger L, Tillman CM, Rodriguez A, Bohlin G. Congnitive functioning and family risk factors in relation to symptom behaviors of ADHD and ODD in adolescents. J Atten Disord 2012;16:284-294.

25. Anastopoulos AD, Smith TF, Garrett ME, Morrissey-Kane E, Schatz NK, Sommer JL, et al. Self-regulation of emotion, functional impairment, and comorbidity among children with ADHD. J Atten Disord 2011;15:583-592. 